\title{
A Simulation for a Predator to Pursuit a Prey Based on Energy
}

\author{
Jae Moon Lee \\ Department of Multimedia Engineering, Hansung University \\ jmlee@hansung.ac.kr
}

\begin{abstract}
For the predator-prey model, most conventional researches have focused on the food chain. In recent computer game and movies, simulation of natural phenomena has been increased. Realistic simulation is a key to the simulation of natural phenomema. It also comes in as an important role when simulating predator-prey chasing Various factors must be put into consideration - such as physical condition, emotion af the predator, and the environment of the pursuit - when creating a realistic simulation of predator-prey chasing. It is not easy to simulate by reflecting all factors independently. Thus, this paper assumes that these independent variables are converted in terms of energy focthe pursuit. It will simulate the situation of a predator chasing a prey using the "energy for the pursuit". To do this, we proposed the consumed energy by using steering forcebased on Newton's law and the needed energy based on physics theory for a predator to move a distance as maximum velocity. A number of experiments were condueted in orden to show the correctness of the proposed energies. Through experiments, this paper illustrates that the proposed energy models are well suited for natural pursuit
\end{abstract}

Keywords: information technology, simulation, agents, predator, prey, energy, force

\section{Introduction}

Flocking is the collectivehavior of certain agents that move accordingly to speed and create a gathering Birds that travel together in the sky, herds of animals moving across the land, fish that swim through the ocean are all valid examples of flocking [1-4, 12]. These collective groups are often targeted as a prey of the predator. Generally, preys form a groupas a mean of avoiding attack of a predator. These groups often show great bond, and act together when confronting a predator This collective behavior has been used in movies and computer games in order to create a more vivid, lively scene. If heterogeneous flocking, which is often easily observed in nature, is introduced in movies and computer games, it will create a far more realistic and rich content. Also, because many researches require visualization of various scientific data, it can be applied to these data visualization depending on time. For instance, it can be utilized in simulating the process of separating impurities from liquid mixtures [6]. It can also be used in clustering massive amounts of documents, which is a kind of data mining $[5,9$, $11]$.

These collective models are mathematical models according to biological intuition that simulate the animation of agent groups. In these models each agent determines the movement based on the environment surrounding it and certain rules of which they counteract with their neighbors in their group. Agent based models interact with other agents depending on their location $[1,14,15]$. In this model, generally law of force is applied; force of attraction (cohesion) and force of repulsion (separation). Cohesion is 
the force that keeps the agents together $[7,8,10]$. When a agent finds itself far away from other agents, it moves towards the group. Each agent moves towards the others to form a group. It keeps them from "merging" into one agent. Each agent looks for the near-by agents. Then it finds the center of the group, that is, the average of the positions. The agent steers towards the center position. Separation is similar to Unaligned Collision Avoidance. This behavior keeps the agents a certain distance away from others $[7,8,10,16]$. This keeps the agents from colliding and crowding to close together. To calculate separation, check each agent against the other agents. If the agents are too close, they repel it using a force based on how close together the agents are. Each agent adds up all the repelling forces to create the steering force. Other forces such as momentum, and the force to avoid obstacles have been suggested as well. Additionally in flocking, force of alignment is used in order to keep homogeneous agents together $[10,12]$. These models have been applied successfully in simulating groups that can be observed in nature. These studies have also been used in solving problems such as pedestrian simulation $[8,12]$.

The model used in this paper is basically the energy model between the predator and the prey based on conventional studies. A predato is an organism that eats another organism. The prey is the organism which the predator eats. Some examples of predator and prey are lion and zebra, bear and fish, and fox and rabbit. The words "predator" and "prey" are almost always used to mean only animals that eat animals, but the same concept also applies to plants: Bear and berry, rabbx and lettuce, grasshopper and leaf. Predator and prey evolve together. The prey is part of the predator's environment, and the predator dies if it does not get cobd, so it evolves whatever is necessary in order to eat the prey: speed, stealth, camouflage, a good sense of smell, sight, or hearing to find the prey, immunity to the prey's poison poison to kill the prey the right kind of mouth parts or digestive system, etc. Likewise the predator is part of the prey's environment, and the prey dies if it is eaten by the predator, so it evolves whatever is necessary to avoid being eaten: speed, camouflage to hide from the predator, a good sense of smell, sight, or hearing to detect the predator, thorns, poison to spray when approached or bitten, etc.

Generally a predator wil- continuously aim to have a chance to chase a prey and a prey will maintain a safe distance from a predator for avoiding it [17]. However if a predator has enoughenergy to chase a certain amount of distance, it will begin to attack a prey. In this case, both predator and prey will apply force to reach a maximum velocity. So they need energy for those forces. This paper focuses on modeling the energy needed for the predator to pursue the prey. The system environment assumed in this paper is a two-dimensional infinite Descartes plane that represents the Earth's surface because this paper considers only a predator and a prey on the earth. This environment can easily be transferred into a three-dimensional environment such as the ocean where fish swim, and the sky where birds fly around. To simplify the problem, the system environment assumes that only 2 agents - the predator and the prey - exist. The friction of the surface is considered to the only interaction between the system environment and the agents. For modeling of further complicated system environments, trees and wind can also be included. This paper represents $i$ as a predator, and $j$ as a prey. The notations, $m_{x}, p_{x}, v_{x}$, and $a_{x}$ represent the current mass, location, velocity, acceleration of agent $x$ respectively. Also, the mass, energy, and time mentioned in this paper are scalar and the location, velocity, and force are vector. In order to simplify the expression, the notation of vector will be omitted. 
Chapter 2 will introduce the modeling of steering forces and the consumed energy of movement. Chapters 3 will analysis on the energy model of a predator and a prey based on physical theory. Chapter 4 will confirm how the energy model suggested in the Chapter 3 operates correctly through an experiment. Chapter 5 will discuss the conclusion.

\section{Steering Forces and Energies}

\subsection{Basic steering forces}

The model in this paper consists of two types of agents: predator and prey. The environment is a two-dimensional infinite Cartesian plane that represents the surface of the earth. Three dimensional environments, such as the sky for birds or the water for fish, usually contain the most stunning swarming behavior. There is assumed to be no interaction between the agents and the environment except for uniform fyiction.

The prey also maintains a certain distance from the predator alerted of its foe. When the predator begins to attack against the prey, the prey runs away to the opposite direction of the predator with the maximum force. Thus, the predator applies pursuit force - a kind of attraction force and the prey applies flee force - a kind of repulsion force. The most basic forces of the agent's aytomatic control force are seek and flee [1, $14,16]$. The seek force is the force that adyances with maximum force towards a target. This force is expressed as the gap between the wish velocity and current velocity in [1, $3]$. The flee force is the opposite of the seek force, The seek force $f_{s}(i, p)$ and flee force $f_{f}(i, p)$ of agent $i$ to target $p$ are the followings not regarding the friction with the surface.

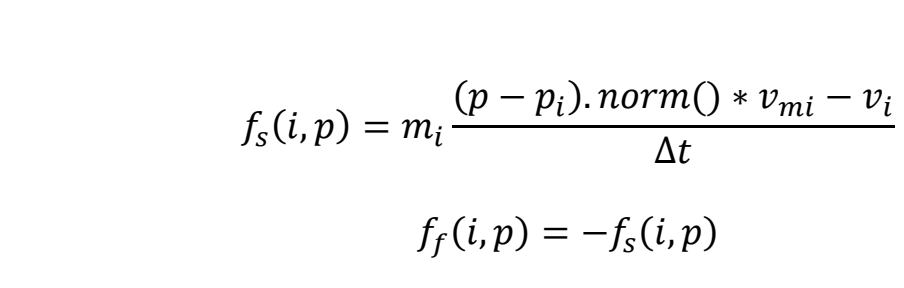

$\left(p-p_{i}\right) \cdot \operatorname{norm}()$ refer $\mathrm{tg}$ the normalization of vector $p-p_{i} . \Delta t$ is the time required for force $f_{s}(i, p)$ to be applied. Directly using seek force as a control force for the predator to chase the prey is not appropriate because the prey is not fixed as a target but is moving. For effective chasing, the most of systems use a method where predicts the location of the prey after time $t$ and then chases the predicted location rather than the current location [3]. There are various considerations to estimate the amount of time $t$. Because more amount of time $t$ will be needed if the predator is far away from the prey, the method in this paper for estimating amount of time $t$ is set in direct proportion with the distance between the two agents. The following (3) is the chase force needed when agent $i$ chases agent $j$.

$$
f_{p}(i, j, \Delta t)=f_{s}\left(i, p_{j}+v_{j} \times t^{\prime}, \Delta t\right)
$$

where $t^{\prime}$ is an amount of time $t$ which is in direct proportion with the distance between the two agents. This paper uses (3) to estimate energy of the predator needed in chasing the prey. When a predator begins to chase a prey, the prey will run away from the predator, and also anticipate the predator's future location while doing so. Thus the equation (5) is the flee force needed when agent $j$ flees from agent $i$. 


$$
f_{e}(j, i)=f_{f}\left(j, p_{i}+v_{i} \times t^{\prime}\right)
$$

\subsection{Measurement of consumed energy of a predator and a prey}

Generally when the predator pursuits the prey, it will check its energy on a certain basis periodically and will decide whether it will continue the chase or cease [17]. For these type of decisions to be made in the simulation, the consumed amount of energy must be measured on a certain basis and must be deducted from the energy it currently owns. In order to measure the consumed energy, the chasing distance can be divided into several small distances as shown in Figure 1.

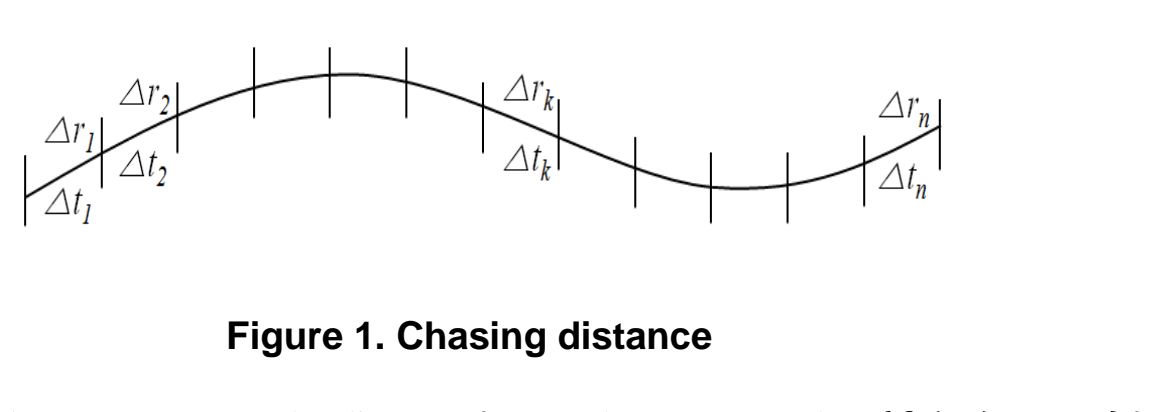

The consumed energy to move the distance $\Delta r_{k}$ can be represented as $\left\{f_{p}(i, j)+m_{i} \mu\right\} \Delta r_{k}$, where $f_{p}\left(i, j, \Delta t_{k}\right)$ is the chase force of $(3)$ and $\Delta t_{k}$ is the time to move the distance $\Delta r_{k}$. Thus, total consumed energy is corhputed as following.

\section{Estimation of NeededEnergy of a Predator and a Prey}

\subsection{Force, frictional force and energy}

Kinetic energy is the physical amount that shows the ability to work. Assume that there is the object moving with constant acceleration on the frictional plane. If force is applied to an object in the moving direction, the velocity of the object will gradually increase Kmetic energy is the physical amount needed when an object is moved to a certain distance in constant acceleration and is defined as the following.

$$
\frac{d E}{d r}=F=m a+m \mu
$$

In (6), $m a$ refers to Newton's force, $\mu$ refers to the coefficient of friction, and $r$ refers to distance. The equation (6) means that in order to move $d r$ with a certain amount of force, there must be $d E$ amount of energy. If both sides of it were integrated, $E$ is calculated as follows.

$$
E=\int_{r_{o}}^{r}(m a+m \mu) d r=\int_{r_{o}}^{r} m \frac{d v}{d r} d r+m \mu \Delta r
$$




$$
\begin{aligned}
& =\int_{v_{o}}^{v} m \frac{d r}{d t} d v+m \mu \Delta r=\int_{v_{o}}^{v} m v d v+m \mu \Delta r \\
& =\frac{1}{2} m\left(v^{2}-v_{o}^{2}\right)+m \mu \Delta r
\end{aligned}
$$

Here, $r_{o}$ and $v_{o}$ refer to the initial location and velocity respectively. $\Delta r$ refers to the distance difference $r-r_{o}$. Thus, the amount of energy needed for mass $m$ to move distance $\Delta r$ while also reaching a velocity of $v$ from the initial velocity of $\mathrm{v}_{\mathrm{o}}$ is same as (7).

\subsection{Estimation of needed energy for a predator}

Generally, the predator will attempt an attack only whên it has enough energy. During chasing the prey, the predator will consume all of the force to reach the maximum velocity. Therefore the predator will peed energy to genetate this force, and the prey will need a minimum amount of energy to flee from the predator's attack. In order to compute the energy for a predator to chase a prey, we assume that the energy of a prey is not limited. That is, a prey has infinite energy so that it can flee until amount of any distance.

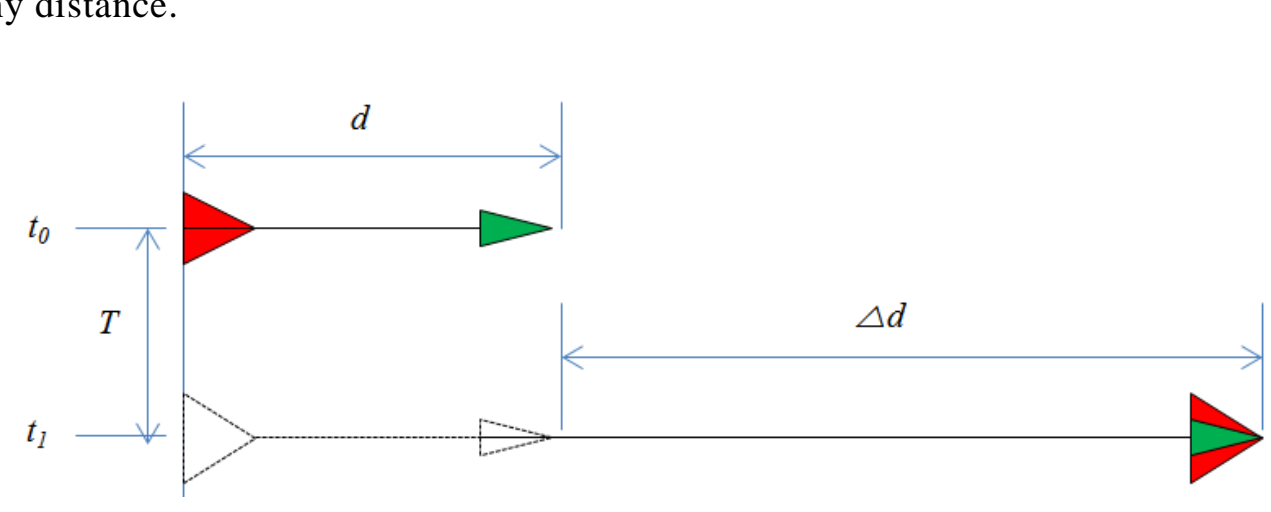

Figure 2. Distance between predator and prey and moving distance

As described in Figure 2, initially the distance between the predator and the prey is $d$. Assume that the - predator successfully capture the prey after it moves the distance $d+\Delta d$. Both agents Will move at their maximum velocity. Let the predator be named as agent $i$ and the prey be agent $j$, and also the agents reach their maximum velocity from the initial velocities, and then the following equation is established.

$$
\begin{aligned}
\Delta r & =d+\Delta d \\
& =\frac{v_{o i}+v_{m i}}{2} t_{m i}+v_{m i}\left(T-t_{m i}\right) \\
& =\frac{v_{o j}+v_{m j}}{2} t_{m j}+d+v_{m j}\left(T-t_{m j}\right)
\end{aligned}
$$

In (8), $T$ is the total amount of time needed in the pursuit, that is, $t_{1}-t_{0}$ in Figure 2. $v_{\text {ox }}$, $v_{m x}$, and $t_{m x}$ represent the initial velocity, maximum velocity, and time consumed in 
reaching maximum velocity of agent $x$ respectively. In the above equation, the second line means the moving distance of the predator, while the third line is to sum the distance $d$ and the moving distance of the prey. Since $v_{o i}, v_{o j}$ refer to the velocity just before chase and flee, the value can be set to 0 . If the value is set as 0 , the equation (8) is like the following.

$$
v_{m i} T=d+v_{m j} T+\frac{v_{m i} t_{m i}-v_{m j} t_{m j}}{2}
$$

In (9), the last term refers to the distance gap of the two agents before they reach their maximum velocity. Let $\beta$ be the difference between $t_{m i}$ and $t_{m i}$ such as $t_{m i}-t_{m}$ 个 Then, $T$ can be simplified as following.

$$
T=\frac{d}{v_{m i}-v_{m j}}+\frac{t_{m i}}{2}+\frac{\beta v_{m j}}{2\left(v_{m i} j v_{m j}\right)}
$$

By using (8) and (10), the value of $\Delta r$ can be computed as following.

$$
\Delta r=\frac{v_{m i} d}{v_{m i}-v_{m j}}+\frac{\beta v_{m i} v_{m j}}{2\left(v_{m i}-v_{m j}\right)}
$$

Finally, based on (7) and (11), the amount of energy needed to chase the prey - $E_{p}(i, j)$ will be like the following.

$$
E_{p}(i, j)=\frac{1}{2} m_{i}\left(v_{m i}^{2}-v_{o}^{2}\left(+m_{i} \mu \frac{v_{m i} d}{v_{m i}-v_{m j}}+m_{i} \mu \frac{\beta v_{m i} v_{m j}}{2\left(v_{m i}-v_{m j}\right)}\right.\right.
$$

In (12), we can assume that the predator already learned $m_{i}, v_{m i}, v_{m j}, t_{m}$ through experience and thys the variables are only $d$ and $u$. The equation (12) is in general. For instance if the current velocity $\left(v_{o i}\right)$ becomes very fast and thus makes $\left(v_{m i}^{2}-v_{o i}^{2}\right)$ smaller, less amount of energy will be needed. Also the amount of energy needed will increase according to the distande between the predator and prey, which is mentioned in the second term in (12). If the, gap between the maximum velocity of the two agents is similar, more time will be consumed in the chase and will result in need of more energy - this also is included the second term in (12).

\subsection{Estimation of needed energy for a prey}

In similar method for the predator, we can compute the minimum energy for a prey to escape from a predator. In order to survive in fleeing, the prey should move at least $\Delta d$ in Figure 2. That is, it means that the prey must have enough energy to move the distance $\Delta d$ with maximum velocity from stopping state.

The minimum energy for a prey to flee from a predator can be induced from (7) by substituting $\Delta d$ to $\Delta r$. And also $\Delta d$ can be calculated from $\Delta r-d$ in (11) as following.

$$
\Delta d=\Delta r-d=\frac{v_{m j} d}{v_{m i}-v_{m j}}+\frac{\beta v_{m i} v_{m j}}{2\left(v_{m i}-v_{m j}\right)}
$$


As replacing $\Delta d$ in (13) to $\Delta r$ in (7), we compute the minimum energy for a prey to escape from a predator as following.

$$
E_{f}(i, j)=\frac{1}{2} m_{j}\left(v_{m j}^{2}-v_{o j}^{2}\right)+m_{j} \mu \frac{v_{m j} d}{v_{m i}-v_{m j}}+m_{j} \mu \frac{\beta v_{m i} v_{m j}}{2\left(v_{m i}-v_{m j}\right)}
$$

Because the prey should move at least $\Delta d$, it must have at least the energy induced in (14). However, the energy in (14) is proportional to the distance $d$ between the predator and the prey. It is correct because (14) is induced under assumption that the predator has already the energy in (12). That is, if the distance $d$ between the predator and the prey is large, then the predator has more energy as shown in (12). Consequently it makes the prey have hore energy.

\section{Experiments}

A number of experiments were conducted in order to show the correctness of the proposed energies. An objective of experiments is to compare the needed energy (12) with the consumed energy (5). Even if the energy of a prey is giyen in (14), we assume that the prey has infinite energy. This assumption is valid because the predator may selectively chase or does not chase the prey but the prey must not only passively make it's movement according to decision of the predator but also must be more desperate than the predator. The experiments were executed according the procedure shown in Figure 3. To simplify the expression, the moment of capture of a prey was omitted.

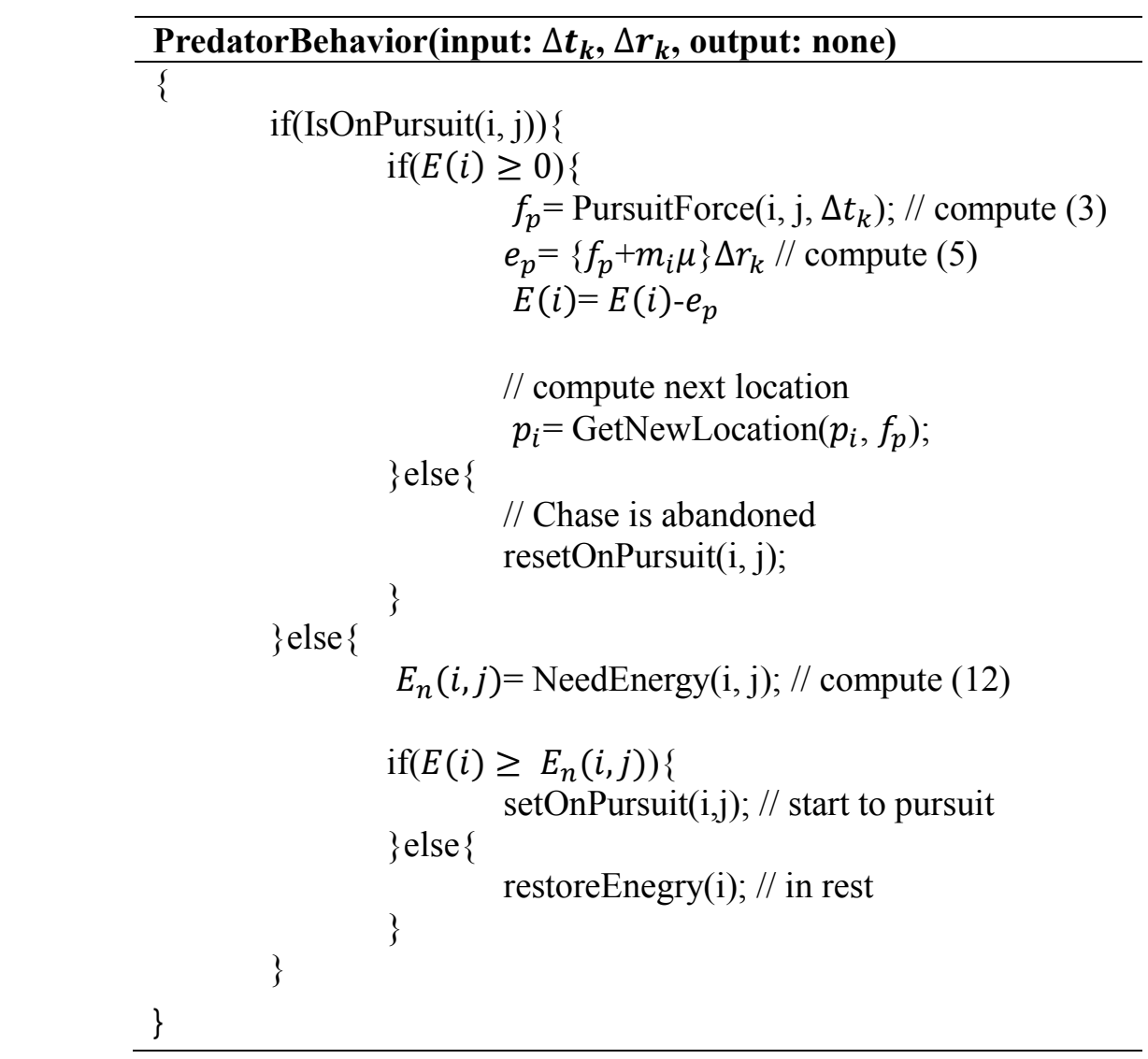

Figure 3. Procedure to control predator's energy 
For the experiments, Windows 7 was used as the operating system and $\mathrm{C}++$ was used as a programming language. Also, algorithms for "seek", "flee", "pursuit", and "evade" was used those suggested in $[3,16]$. The experiment focuses on how much the needed energy, (12), based on physical theories is coincided to the consumed energy, (5), based on simulations of "seek", and "pursuit".

The size $\left(s_{i}\right)$, mass $\left(m_{i}\right)$, maximum velocity $\left(v_{m i}\right)$ of a predator were each set to 5 pixels, $5 \mathrm{~kg}$, and 10 pixels $/ \mathrm{sec}$ respectively. The size, mass, maximum velocity of a prey were each set as $60 \%$ of those of the predator. Under the mentioned circumstances, the needed energy and the actual consumed energy were calculated with a variable of distance between the predator and the prey and friction by performing the procedure of Figure 3. It is not easy to estimate the value of $\beta$ in (11). This is because it is not easy to estimate the time $t_{m x}$ that takes for an agent $x$ to reach max velocity For those reasons, this paper has set $\beta$ as 0 for calculation.

Table 1. $E_{n}(i, j)$ and $E_{c}(i, j)$ versus distance

\begin{tabular}{|c|c|c|c|c|c|}
\hline distance & $16 s_{i}$ & $32 s_{i}$ & $64 s_{i}$ & $128 s_{i}$ & $256 s_{i}$ \\
\hline$E_{n}(i, j)$ & 14250 & 28250 & 56250 & 112250 & 224250 \\
\hline$E_{c}(i, j)$ & 15558 & 29561 & 57558 & 13559 & 225564 \\
\hline delta & 1308 & 1314 & 1308 & 1309 & 1314 \\
\hline
\end{tabular}

Table 1 shows the consumed ehetgy and the needed energy based on the distance between the predator and the presk. The coefficient of friction was set to 7.0. In Table 1, $s_{i}$ refers to the size of the predator. Thus, $32 s_{i}$ means that the distance between the predator and prey is 32 cimes the size of the predator. The collected data in Table 1 shows, regardless of distance, that $E_{n}(t, j)$ is deficient as much as about 1310 . This is because the $\beta$ value has been set to 0 . The $\beta$ value in (12) is independent of $d$, and thus the value of deta in Table 1 is nearly constant. The data in Table 1 shows that as the distance increases, $E_{n}(i, j)$ and $E_{c}(i, j)$ increases as well. This is the obvious result according to (12) and (5).

Table 2. $E_{n}(i, j)$ and $E_{c}(i, j)$ versus coefficient of friction

\begin{tabular}{|c|c|c|c|c|c|}
\hline$\mu^{\prime}$ & 1 & 3 & 7 & 13 & 19 \\
\hline$E_{n}(i, j)$ & 8250 & 24250 & 56250 & 104250 & 152250 \\
\hline$E_{c}(i, j)$ & 8355 & 24756 & 57560 & 106778 & 155988 \\
\hline delta & 105 & 506 & 1310 & 2528 & 3738 \\
\hline
\end{tabular}

Table 2 is the measurement of the needed energy and the consumed energy according to the coefficient of friction. The distance between the predator and the prey has been set to $64 s_{i}$, and the coefficient of friction is a variable that varies through 1 to 19 . As shown in Table 2, the needed energy and the consumed energy are increasing along as the coefficient of friction increases. This also is because (12) and (5) are in direct proportion with the coefficient of friction $\mu$. Contrary to Table 1 , the value of the delta increases as the value of $\mu$ varies in Table 2. This is because the $\beta$ value is in direct proportion with $\mu$ value in (12). Regarding the results of Table 1,2 , it is plausible to conclude that the consumed energy of (5) which was based on "seek" and "pursuit" and 
the needed energy of (12) which was based on physical theories were correctly modeled.

Finally, Table 3 shows the relationship between the needed energy and the consumed energy according to the value of $\beta$, where the value of the distance and the coefficient of friction are fixed as $16 s_{i}$ and 7.0 respectively. Because $E_{c}(i, j)$ is independent on the value of $\beta$, its values must be constant. But the values of $E_{c}(i, j)$ in Table 3 are to be measured, not to be calculated, and so they are nearly constant. However, the fact that $E_{n}(i, j)$ does not exactly same to $E_{c}(i, j)$ is because $E_{c}(i, j)$ is calculated as the unit of $\Delta t_{k}$.

Table 3. $E_{n}(i, j)$ and $E_{c}(i, j)$ according to $\beta\left(=t_{m i}-t_{m j}\right)$

\begin{tabular}{|c|c|c|c|c|c|c|}
\hline$\beta$ & 0 & 1 & 2 & 4 & 8 & 16 \\
\hline$E_{n}(i, j)$ & 8250 & 8400 & 8550 & 8850 & 9450 & 10650 \\
\hline$E_{c}(i, j)$ & 8957 & 8957 & 8957 & 8956 & 895 & 8956 \\
\hline delta & 707 & 557 & 407 & 106 & 493 & -1694 \\
\hline
\end{tabular}

\section{Conclusions}

In the environment of predator-pre $\vec{y}$. most of the researches are to study the food chain, while this paper is to study of energy of a predator and a prey when a predator pursuits a prey. In order to chase a prey, a predator should save its energy so that it can move some distance. And aiso d prey should reserve always the minimum energy in order to avoid attack of a predator. Thus realistic simulation for a predator to chase prey should be executed based on those energies. In order to do this, we simplified the environment of the problem such as ony a predator and a prey allowed and introduced the method to measure the consumed energy based on steering forces. And then we proposed the method to estimate the needed energy for a predator and a prey based on physical theory so that a predator can decide by considering its energy whether it chase the prey or does not. Through experiments, the paper has shown that the suggested energy models are well suited for natural pursuit. Further research will focus on environments where various predators and preys coexist. Thus, in an environment where multiple predators and preys exist, the choice criterion of the predator and cooperation/of predators will be researched.

\section{Acknowledgements}

This research was supported by Basic Science Research Program through the National Research Foundation of Korea(NRF) funded by the Ministry of Education, Science and Technology(2012R1A1A2001091).

\section{References}

[1] C. W. Reynolds, "Flocks, Herds, and Schools: A Distributed Behavioral Model", SIGGRAPH, vol. 21, no. 4, (1987), pp. 25-34.

[2] I. D. Couzin, J. Krause, R. James, G. D. Ruxton and N. R. Franks, "Collective Memory and Spatial Sorting in Animal Groups", J. theory Biol., (2002), pp. 1-11.

[3] M. Buckland, "Programming Game AI by Example", ISBN 1556220782, Wordware Publications, (2005).

[4] R. Olfati-Saber, "Flocking for Multi-Agent Dynamic Systems: Algorithms and Theory", IEEE Trans. On Automatic Control, vol. 51, no. 3, (2006), pp. 401-420. 
[5] X. Cui, J. Gao and T. E. Potok, "A Flocking Based Algorithm for Document Clustering Analysis", Journal of System Architecture, Special issue on Nature Inspired Applied Systems, (2006), pp. 505-515.

[6] W. Zheng, J. -H. Yong, J. -C. Paul, "Visual Simulation of Multiple Unmixable Fluids", J. Comput. Sci. Technol., vol. 22, no. 1, (2007), pp. 156-160.

[7] M. Ballerini, N. Cabibbo, R. Candelier, A. Cavagna, E. Cisbani, I. Giardina, V. Lecomte, A. Orlandi, G. Parisi, A. Procaccini, M. Viale and V. Zdravkovic, "Interaction ruling animal collective behavior depends on topological rather than metric distance: Evidence from a field study", In Proceedings of the National Academy of Sciences, vol. 105, no. 4, (2008), pp. 1232-1237.

[8] I. Karamouzas, J. Bakker and M. H. Overmars, "Density Constraints for Crowd Simulation", International IEEE Consumer Electronics Society Games Innovation Conference, (2009), pp. 160-168.

[9] G. Folino, A. Forestiero and G. Spezzano, "An adaptive flocking algorithm for performing approximate clustering", Inf. Sci., vol. 179, no. 18, (2009), pp. 3059-3078.

[10] S. Kim, C. Hoffmann and J. M. Lee, "An Experiment in Rule-based Crowd Behavior for Intelligent Games", In Proceedings of Fourth International Conference on Computer Sciences and Convergence Information Technology, (2009), pp. 410-415.

[11] G. Folino, A. Forestiero and G. Spezzano, "An adaptive flocking algorithm for pe forming approximate clustering", Inf. Sci., vol. 179, no. 18, (2009), pp. 3059-3078.

[12] J. M. Lee, "An Efficient Algorithm to Find k-Nearest Neighbors in Flocking Bellavior", Information Processing Letters, vol. 110, no. 14-15, (2010), pp. 576-579.

[13] X. Luo, S. Li and X. Guan, "Flocking algorithm with multi-target tracking for multi-agent systems", Pattern Recognition Letters, vol. 31, no. 9, (2010), pp. 800-805.

[14] V. Zhdankin and J. C. Sprott, "Simple predator-preyswarming model", PHYSICAL REVIEW, vol. E 82, (2010), pp. 056209-1 7.

[15] D.-h. Cho, Y. h. Lee, J. H. Kim, S.-Y. Park and D.W. Rhee, "XPC Control Model for Defense in Soccer Game Applying the Decision Tree Learning Algorithm", Journal of Korea Game Society, vol.11, no. 6, (2011), pp. 61-70.

[16] S. H. Cho and J. M. Lee, "Group Behavior Simulation of Multi-Agents by Using Steering Forces in an Enclosed Space", Journal of Korea Game Society, vol. 11. nø. 1, (2011), pp. 111-120.

[17] J. M. Lee, "An Evaluation of Pursuing Energy fon Predator", Proceedings of the 7th European Computing Conference, (2013), pp. 368-372.

[18] S. Kim, J. M. Lee, V. Ramachandran and S. Chin, A biological simulation game using Prey-Predator model", Information - An international interdiscipli)ary,journal, vol. 4, no. 16, (2013), pp. 2607-2618.

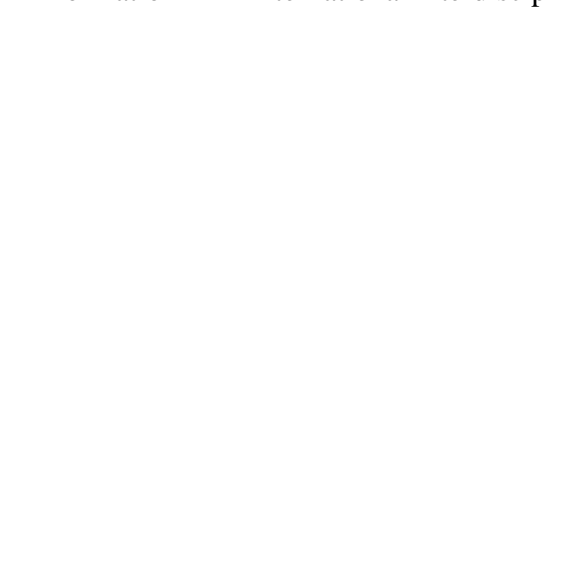

